\title{
Development of a rhythmic auditory biofeedback system to assist improving the kinetic chain for bat swing performance
}

\author{
Teppei Onishi ${ }^{*}$, Kazuhiro Yasuda$^{2}$, Suguru Kawata ${ }^{1}$ and Hiroyasu Iwata ${ }^{1}$
}

\begin{abstract}
Baseball has been enjoyed by many generations and there are many people who play the game. Swing speed increases are generally required to develop ideal batting skill; nevertheless, no study has proposed a system to swing speed and develop an idea swing performance kinetic chain using auditory biofeedback, which is often used for motor modification. Thus, the purpose of our study was to devise a system that allows users to develop their kinetic chains and increase swing speeds using auditory cues, and to verify the proposed system's effect in the bat swing performance of novices. We developed a system that output auditory cues (i.e., sound) linked with body motion. First, we conducted an experiment to confirm whether our proposed method could detect the difference between skilled players and beginners. Second, we applied the system to a tee-batting task in 22 novice baseball players. Using our proposed system, participants could increase swing speed and acquire a better kinetic chain in upper body motion but not in trunk motion. We expect this system is, in part, applicable to developing swing performance that requires sequential motion. This auditory biofeedback system warrants further investigation.
\end{abstract}

Keywords: Sports engineering, Baseball, Swing performance, Auditory biofeedback, Kinetic chain, Motor learning

\section{Background}

Batting is one of the most important baseball skills [1]. Batters typically need to swing the bat quickly, and increasing swing speed appears to increase batters' hit to home run ratio. To increase swing speed, batters must develop an ideal kinetic chain; however, learning the ideal kinetic chain appears to be difficult for beginners.

Previous research has found that appropriate physical motion leads to increased swing speed and that professional baseball players usually swing with an ideal kinetic link [2]. For instance, professional baseball players usually start rotating their hips before rotating their shoulders, which, in turn, rotate before the arms begin moving [2]. This sequence of movements, known as the "kinetic chain," has been defined as "the mechanical linkages of body segments which allow for the sequential transfer of

\footnotetext{
*Correspondence: bb8.runbird73@akane.waseda.jp

${ }^{1}$ Graduate School of Creative Science and Engineering, Waseda

University, 3-4-1 Okubo, Shinjuku-ku, Tokyo 169-8555, Japan

Full list of author information is available at the end of the article
}

forces and motions when performing a task such as throwing $[3,4]$." enables the distal segment (i.e., wrist) to efficiently accelerate by moving in turn from the proximal segment (i.e., hip), and an ideal kinetic chain can likely induce faster batting speed.

Instruction (i.e., coaching) is a typical training method for increasing swing speed and developing the kinetic chain. This is because coaches can confirm swing mechanics in real time and provide instructions for improvement. However, coaches tend to provide qualitative instruction. Thus, it is often difficult to understand coaches' intention because they instruct with vague expressions such as "onomatopoeia" or "mimetic word". In addition, instruction is often based on the coach's experiences. Thus, training quality depends on coaching skill, and training might be unable to help players increase their swing speed and develop an ideal kinetic chain.

Previous research has assessed the kinetic chain using micro-sensors. Ahmadi et al. showed that inertial sensors located on the chest, upper arm, and hand of tennis 


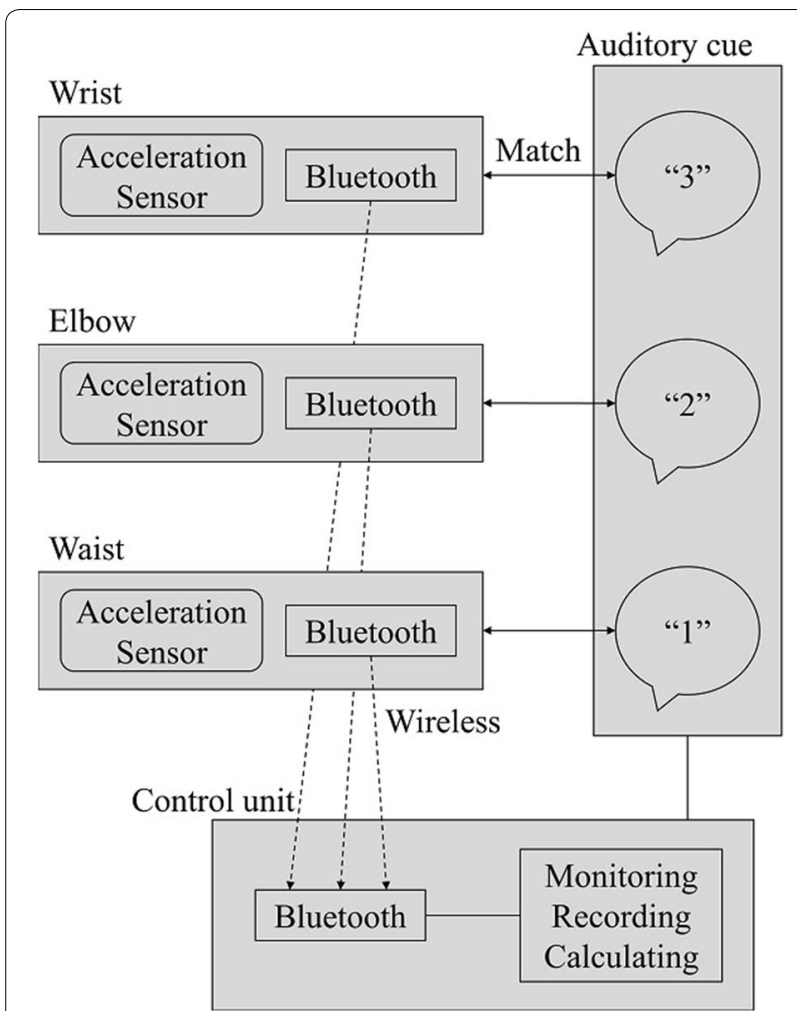

Fig. 1 Configuration diagram

players enabled detection of specific strokes [5]. In addition, research using inertial sensors mounted on players' bodies found differences between skill levels in tennis strokes [6], golf swings [7], and pitching motions [8]. Further, Ghasemzadeh et al. developed a quantitative golf swing model using data from micro-sensors placed on participant bodies and clubs [9]. As for batting motion, Ghasemzadeh and Jafari evaluated swings using sensors placed on the hip, chest, and wrist, and developed a signal processing model that can calculate swing motion sequence and timing using sensor data and discriminate between good and bad swing performance [10]. The authors proposed that the model could be used for training baseball players; however, they did not report how using this model can affect swing performance.

Engineering support for movement correction has mainly been reported in the medical rehabilitation field. For example, previous studies found that patients usually perceive important biofeedback information (i.e., cues or patterns) to modify movements. Ki et al. found that auditory cues during gait can improve weight bearing in hemiplegia patients [11]. Schauer et al. reported that when stroke patients provide musical feedback through heel strikes, their gait is improved [12]. Owaki et al. revealed that auditory feedback conveyed through foot pressure sensors can improve the short-term walking performance of stroke patients [13]. Furthermore, sounds corresponding to footstep rhythms can support patients with gait disturbances [14]. Baram et al. also reported that auditory cues can improve walking speed and stride length in Parkinson's disease patients [15]. In terms of haptic-based biofeedback systems, we previously reported that vibro-tactile cues can improve performance during gait and balance in stroke patients [16-18]. It is assumed that patients can easily correct movement because sensing cues enable them to viscerally perceive their own movement. Thus, it is possible that methods that enable visceral perception are likely to be applicable in sports performance. Only one study reported the application of auditory cues intended to enhance a swimmer's perception of the interaction between their body and surrounding water pressure [19]. However, no study has proposed a system to increase swing speed or develop an ideal kinetic chain using auditory biofeedback. Thus, this study's purpose is to devise a system that helps users develop their kinetic chain and increase swing speed using auditory cues, and to verify proposed system's effect on swing speed. We hypothesize that baseball players could improve their kinetic chains and increase swing speed using auditory cues that provide information on body motion sequence and timing.

\section{Methods}

\section{Overview of the auditory cue biofeedback system}

The auditory cue feedback system consists of three acceleration sensors (TSND151, ATR-Promotions, Japan) that capture motion data during a bat swing. The subject was fitted with these sensor units, with one on the waist, one on the bottom of the elbow, and one on the bottom wrist (Figs. 1 and 2). Acceleration measurements from the sensors were relayed to a PC via Bluetooth. Acceleration measurements were sampled every $80 \mathrm{~ms}$ with a

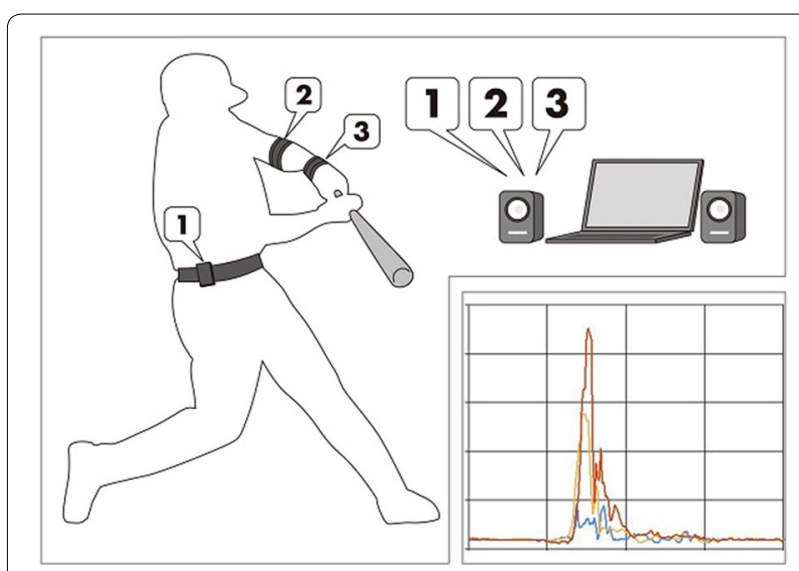

Fig. 2 Overview of the auditory cue biofeedback system 
$10 \mathrm{~ms}$ transmission period. The system also had a screen that displayed acceleration in real time (Fig. 2). When a body part started moving, the system's PC would output an auditory cue. The auditory cues were related to each body part (i.e., waist: one, elbow: two, wrist: three). Thus, if realizing an ideal kinetic chain, the subject would hear a sequenced auditory cue (one $\rightarrow$ two $\rightarrow$ three) after the swing motion. The PC allowed changing of auditory cue volume to deal with noise around training field.

We applied auditory BF for three reasons: (1) auditory feedback is preferable for motor learning [20], (2) visual and haptic feedback tends to interfere with the bat swing and other information, and (3) auditory BF would be an optimal method for instructing temporal sequence (e.g., melody road [21]) especially when swinging a bat. Regarding point (2), we assumed that the players would incorporate kinetic skills in their future training. Visual feedback would detract players from watching the pitching motion and the pitched ball, degrading their hitting accuracy. Meanwhile, haptic feedback would combine with the sense of the swing motion. Increasing the magnitude of the haptic feedback is a poor solution, because large haptic feedback exerts a negative impact on swing motion. Auditory feedback, in contrast, enables the trainees to ascertain their ball pitches (visual sense) and swing motions (somatic sense) without interference.

\section{Auditory cue sound algorithm}

Swinging a bat is a complex motion because it includes trunk rotation and upper limb motion (e.g., arm extension). We thus defined exercise intensity, $a$, which is calculated using the values $a_{x}, a_{y}$, and $a_{z}$, which are captured by the acceleration sensors.

$$
a^{i}=\sqrt{\left(a_{x}^{i}\right)^{2}+\left(a_{y}^{i}\right)^{2}+\left(a_{z}^{i}\right)^{2}} \quad(\mathrm{i}=1,2,3)
$$

We calculated relative exercise intensity $\left(\mathrm{R}^{\mathrm{i}}\right)$ using $a^{i}$. If the $R^{i}$ value was larger than the threshold value (Table 1 ), the auditory cues would sound. We then considered that the relative exercise intensity at the waist is the same as the exercise intensity at waist because it is most proximal body part (Eqs. 2 and 3).

$$
\begin{aligned}
& R^{i}=a^{i} \quad(\mathrm{i}=1) \\
& R^{i}=a^{i}-a^{i-1} \quad(\mathrm{i}=2,3)
\end{aligned}
$$

\section{Experiment to detect difference by skill level}

Before applying our proposed system to novice baseball players, we needed to confirm whether this method could detect the difference between a skilled player and a novice. We conducted an experiment with six young and healthy participants consisting of three skilled players and three beginners (Fig. 3, Table 2). This experiment
Table 1 Threshold value to detect start of motion

\begin{tabular}{llll}
\hline Body part & 1: waist $\left(\mathbf{m} / \mathbf{s}^{\mathbf{2}}\right)$ & 2: elbow $\left(\mathbf{m} / \mathbf{s}^{\mathbf{2}}\right)$ & 3: wrist $\left(\mathbf{m} / \mathbf{s}^{\mathbf{2}}\right)$ \\
\hline Threshold value & 4.9 & 14.7 & 14.7 \\
\hline
\end{tabular}

was performed with approval of the Waseda University Ethical Review Board.

We asked the participants to swing a $0.55 \mathrm{~kg}$ bat as if they were hitting a ball at belt height in the pitcher's direction ten times. We chose these parameters because previous studies found that swing form is affected by ball speed [22], whether a pitch is a breaking ball [23], ball height $[24,25]$, direction the ball is to be hit [26], and the weight of the bat [27]. We then captured acceleration data, which we used to verify the validity of the system's judgment (i.e., difference between skilled and novice). We also calculated the time at which the relative exercise intensity $\left(R_{i}\right.$; see Eqs. $\left.1-3\right)$ exceeded the threshold value (Table 1). Using this time, we judged whether the swing was ideal or non-ideal. We defined an ideal swing as a sequenced motion (i.e., waist $\rightarrow$ elbow $\rightarrow$ wrist) .

\section{Results}

Table 2 shows participant swing states. The ideal swing (i.e., waist $\rightarrow$ elbow $\rightarrow$ wrist) ratio in skilled players was $96.7 \%$, meaning that nearly all the skilled players accelerated in turn (i.e., waist, elbow, wrist) during the swing motion in the experiment. In contrast, the ideal swing ratio in beginners was $10.0 \%$, meaning that beginners did not have as much control over the sequential body motion as skilled players did.

\section{Training program to develop kinetic chain}

As described the first experiment, beginners have difficulty using an ideal kinetic chain when swinging a bat. Therefore, we proposed a training program based on motor learning stages to help develop an ideal kinetic chain [28]. In the first and most important stage for novices, participants trained focusing on only one joint (i.e., waist $\rightarrow$ elbow or elbow $\rightarrow$ wrist). Thus, participants aimed at mastering the motion of each joint first.

We instructed participants to twist their trunks and to start their motion from the waist and move to the elbow in turn. After this instruction, participants listened for auditory cue sounds while swinging the bat. If participants could not realize the ideal cue, they revised their own motion. Participants repeated these practices until they acquired ideal motion. The swing motion from the elbow to the wrist is almost one flow. For upper limb practice, we instructed beginners to flex their elbows. After this first stage, the beginners repeatedly practiced their swing and listened to the auditory BF system to develop more automatic body movements. 
Table 2 Participant characteristics and difference between skill levels detected by proposed system

\begin{tabular}{llllll}
\hline & Age (years) & Height $(\mathbf{c m})$ & Weight $(\mathbf{k g})$ & Baseball career (years) & Good swing (\%) \\
\hline Skilled players $(n=3)$ & $22.7 \pm 0.5$ & $175.0 \pm 5.7$ & $68.3 \pm 5.4$ & $11.7 \pm 0.5$ & $96.7 \pm 4.7$ \\
Novices $(n=3)$ & $24.0 \pm 2.2$ & $173.3 \pm 4.5$ & $66.3 \pm 2.6$ & 0 & $10.0 \pm 14.1$ \\
\hline
\end{tabular}

Mean \pm SD, $n$ number of participants

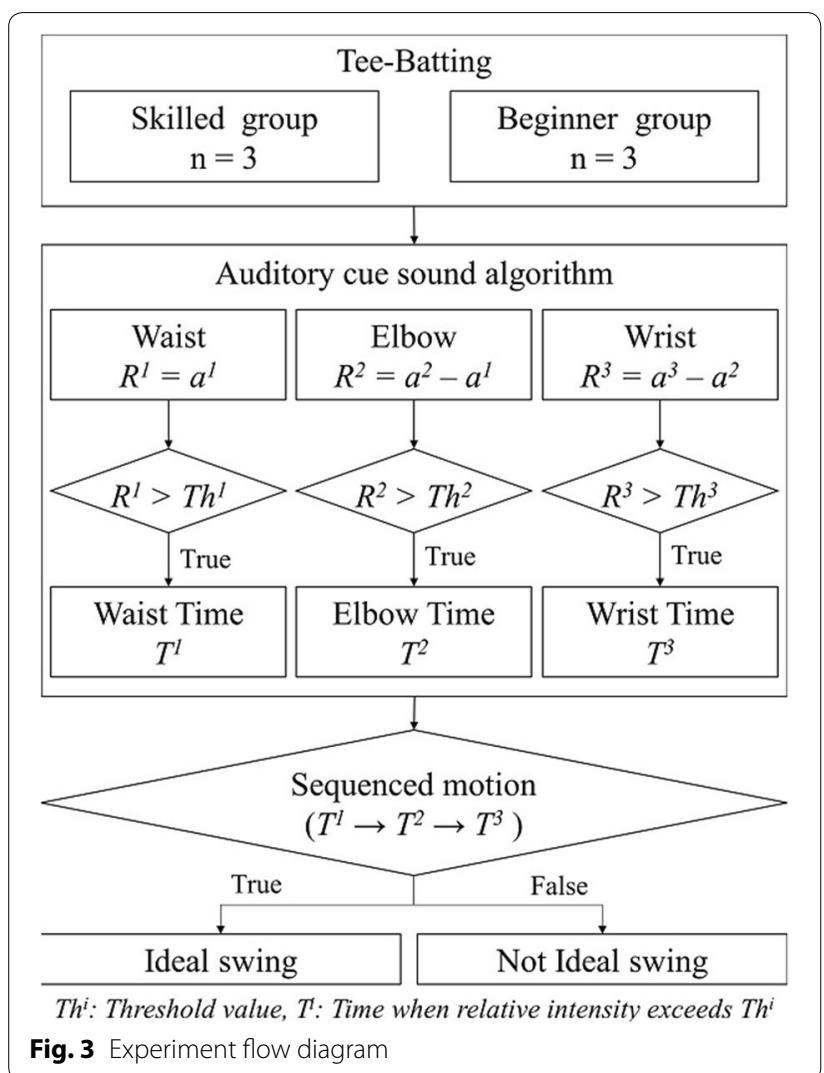

\section{Examination of test validity in baseball beginners Target}

We applied our proposed system to 22 healthy adult beginning baseball players to examine the system's feasibility (Fig. 4). We randomly divided the 22 participants into a training group and a control group. Table 3 shows the characteristics of each group. Baseline performance (i.e., initial swing speed) was not significantly different between the two groups This examination's procedure was approved by the Waseda University Ethical Review Board.

\section{Design}

After warming up and applying sensors from the proposed system, we had participants hit a teed ball at belt height toward a net placed in the pitcher's direction as
Table 3 Participant characteristics

\begin{tabular}{lllll}
\hline Group & Age (years) & Height $(\mathbf{c m})$ & Weight $(\mathbf{k g})$ & $\begin{array}{l}\text { Swing Speed } \\
(\mathbf{k m} / \mathbf{h})\end{array}$ \\
\hline $\begin{array}{c}\text { Training } \\
(\mathrm{n}=14)\end{array}$ & $22.1 \pm 0.9$ & $171.6 \pm 6.3$ & $62.1 \pm 7.6$ & $95.0 \pm 20.7$ \\
$\begin{array}{c}\text { Control } \\
(\mathrm{n}=8)\end{array}$ & $22.8 \pm 1.0$ & $167.1 \pm 11.3$ & $59.6 \pm 10.8$ & $99.8 \pm 25.8$ \\
\hline
\end{tabular}

Mean $\pm S D, n$ number of participants

hard as possible five times. Participants had performed this tee-batting task during the pre-test. Following the pre-test, participants practiced by applying the proposed training program. During training program, training group listened to auditory cue BF, control group didn't. Lastly, participants performed a tee batting post-test during which the training group listened to auditory cue BF.

\section{Evaluation}

During the experiment, we examined participants' swing speed and participants' maximum waist, shoulder, elbow, and wrist acceleration. The first variable shows whether a participant could increase swing speed and bat swing skill, and the second showed whether beginner participant could develop the ideal kinetic chain.

\section{Analysis}

We compared swing speeds in the training and control groups, subtracting pre-test values from post-test. For the kinetic chain, we compared pre-test and posttest acceleration in the training group. We analyzed the data using the Mann-Whitney U test, and the Wilcoxon signed-rank test as a non-parametric test because of the small sample size [29]. We set our significance level at $\mathrm{p}<0.05$.

\section{Results}

\section{Swing performance}

Compared with the control group, swing speed in the training group increased significantly $(\mathrm{p}<0.05$, Fig. 5).

\section{Kinetic chain ability}

In the training group, the post-test maximum acceleration of the elbow and wrist increased significantly 


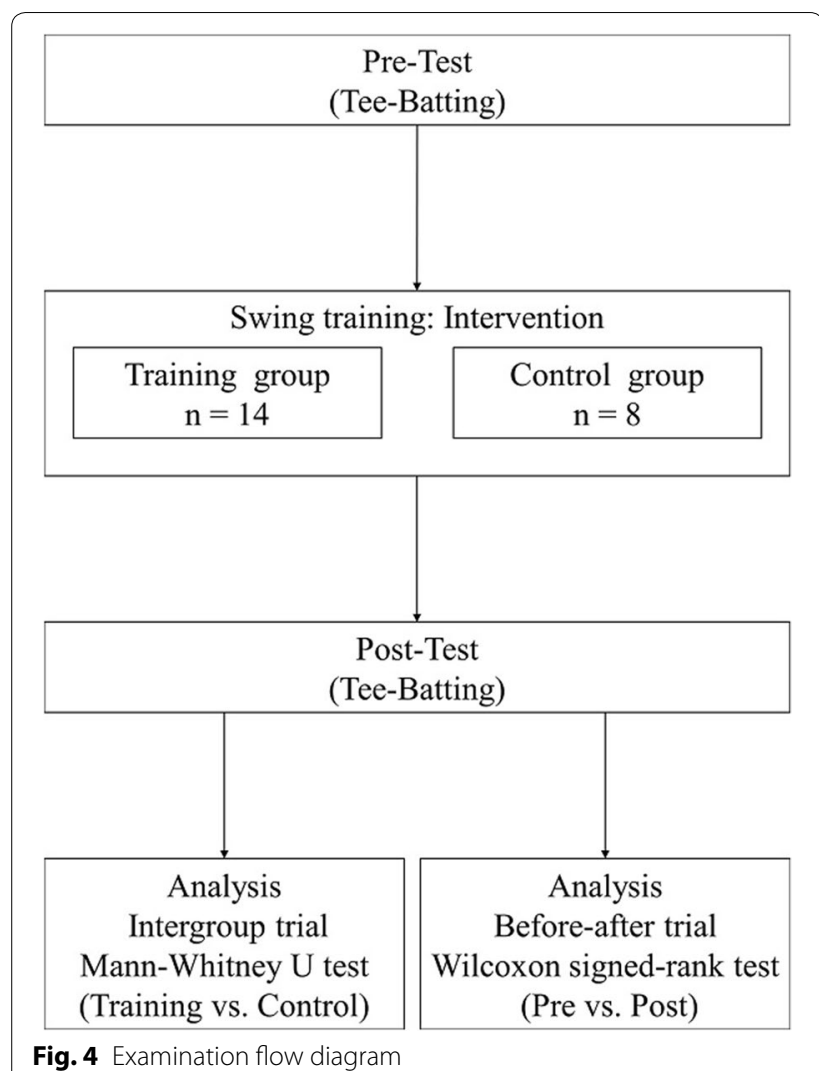

compared to pre-test the pre-test maximum $(\mathrm{p}<0.05$, Fig. 6). However, we found no significant differences between the pre-test and post-test waist and shoulder maximum acceleration.

\section{Discussion}

In this study, we devised a system to increase swing speed and develop an ideal kinetic chain using auditory cues that provide information on the sequence and timing of body motions. We structured an algorithm to calculate relative exercise intensity. We then verified whether this method could detect a difference between skilled and beginning baseball players. Finally, we applied a training program using our proposed system to beginning baseball players. As a result of the training program using our proposed system, participants in the training group had increased swing speeds compared to those of control group participants. Further, players in the training group could develop an ideal kinetic chain in the upper body after the training using the proposed auditory BF system, but not in the trunk. These results suggest that our proposed system is, in part, effective and feasible for training baseball beginners in batting.

In this study, both swing speed and maximum acceleration of the upper body (i.e., elbow and wrist) increased

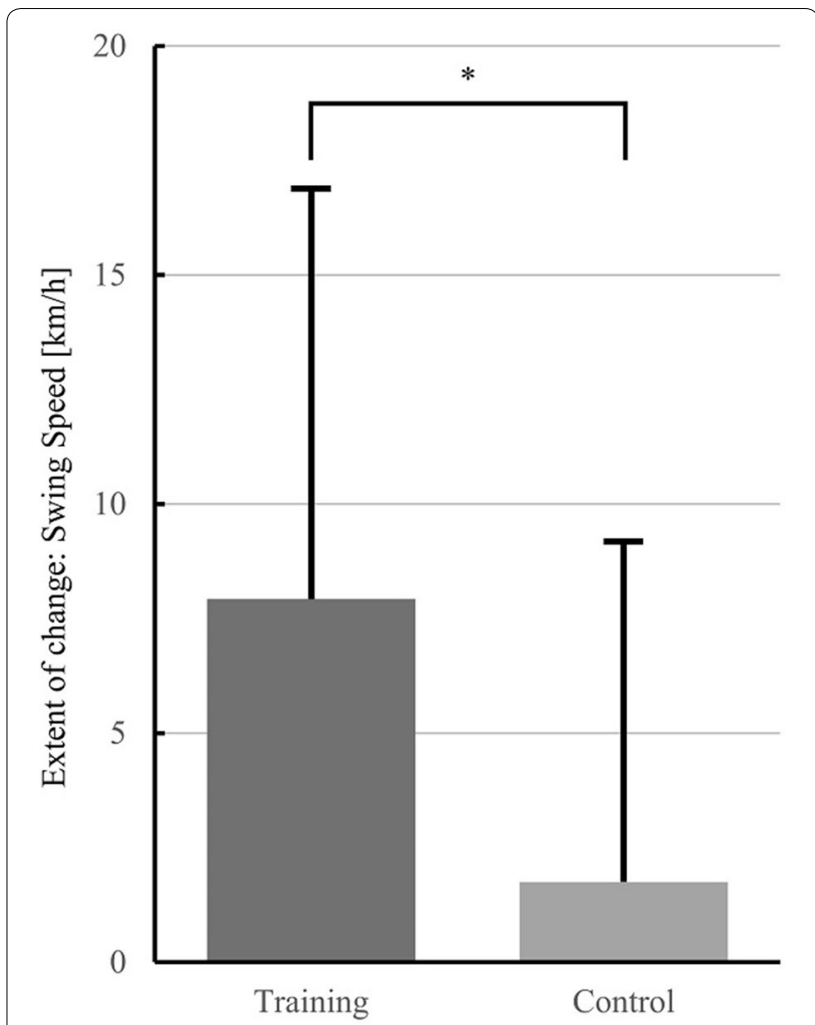

Fig. 5 Variation in the extent of change (pre-test and post-test difference) in swing speed $(\mathrm{km} / \mathrm{h}) .{ }^{*} p$ values were derived using the Mann-Whitney $U$ test $\left({ }^{*} p<0.05\right)$

significantly. This suggests that listening to auditory cues enables participants to enhance their swing skill by developing an ideal kinetic chain in the upper body. Although we cannot clarify the underlying mechanisms, we explore two viewpoints. From the motor-control perspective, we postulate that the feedback-error-learning model [30] functions by auditory feedback. Usually, the players revise their motions based on feedback from their previous motion. However, beginners have insufficient knowledge to acquire the correct feedback, so cannot easily improve their swing performance (i.e., their kinetic chain) (Fig. 7-A). The proposed system provides very simple feedback (a number sequence) that enables beginners to understand their motions, and thus improve their skills (Fig. 7-B). From the motor-learning perspective, we expect that novices can improve their kinetic chain by comprehending what constitutes an ideal motion. At the cognitive stage [28], a learner needs an overall understanding of the skill. In other words, beginners are required to understand the ideal swing and compare it with their own swing. Although these requirements are often learned in previous training and instruction, beginners using the proposed system can 


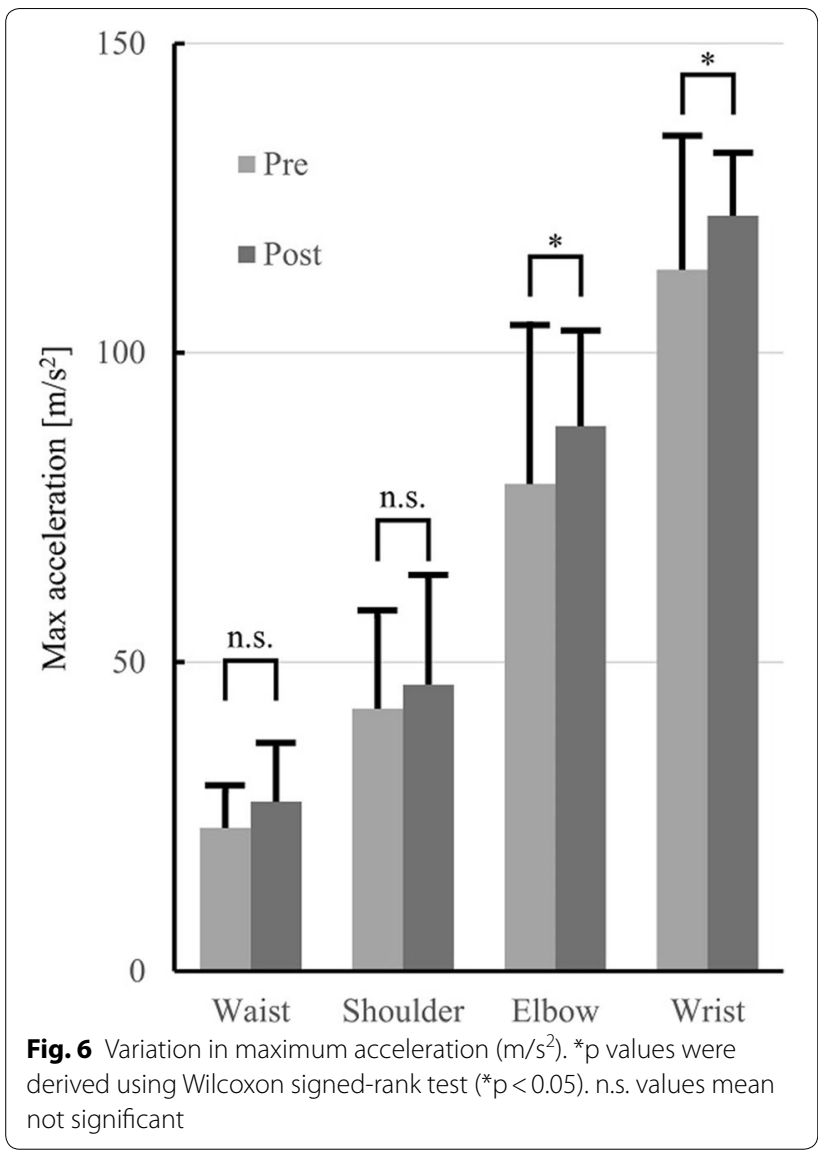

simply understand their own swing through the auditory feedback, and the ideal swing as a number sequence. Thus, beginners might improve their kinetic chain through a visceral cognitive route.

In contrast to upper body motion, maximum acceleration of the trunk (i.e., waist and shoulder) did not significantly increase. As for limitations of the effect, one possible explanation might be mobility differences. Controlling trunk movement might depend on different factors than controlling the upper body, possibly because the trunk's inertia moment seems to be relatively large. In this context, sequential movements from other body parts would be important to move the trunk. Previous studies have reported that lower limb torque is a major contributor to trunk motion [31, 32]. Thus, it is possible that listening to auditory cues was insufficient to develop an ideal kinetic chain in the trunk.

This study has some limitations. First, this feasibility study was conducted using a small group of novice baseball players and we only evaluated the auditory BF system's immediate effects. Therefore, rigorously designed long-term interventional studies are necessary to determine the exact training efficacy of our proposed auditory BF system in novice baseball players. Also, the sampling

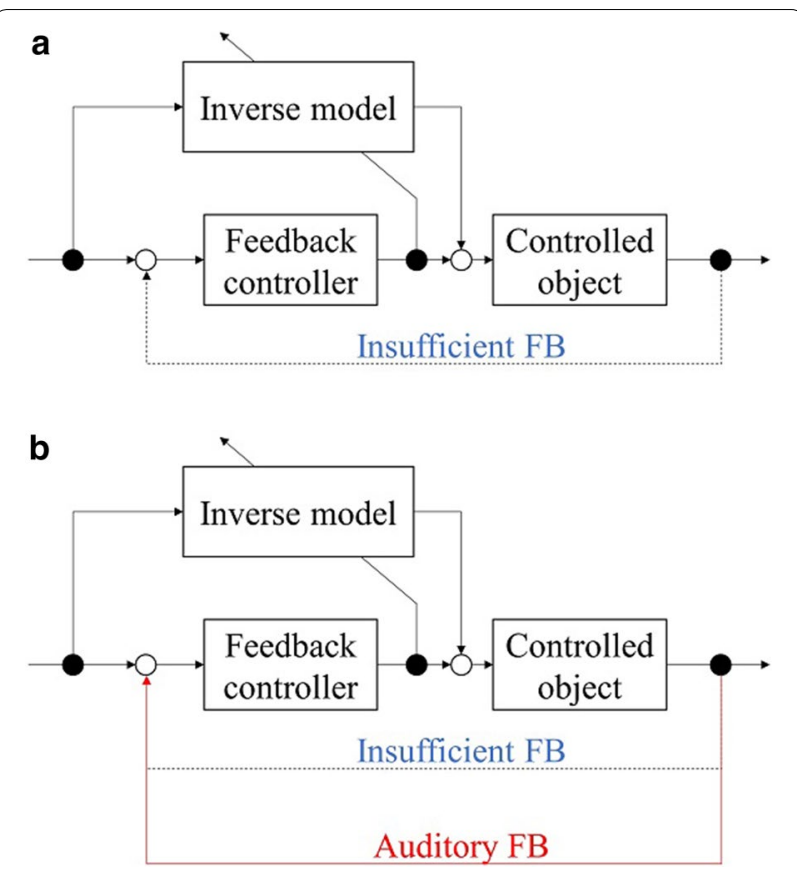

Fig. 7 Feedback-error-learning model [30]. Learning by beginners in the absence $(\mathbf{a})$ and presence $(\mathbf{b})$ of the proposed system

time of the acceleration should be increased for monitoring sports motions. We believed that the sampling time is sufficient because the proposed system detected the difference between a skilled player and a novice. However, acceleration monitoring with a fast sampling time is required for skilled players.

\section{Conclusions}

In this study, we devised an auditory BF system to enable baseball novices to increase their swing speed and develop an ideal kinetic chain. We confirmed the judgment validity of the proposed system. Finally, we applied a developed auditory BF system in 22 novice baseball players to examine the system's feasibility. The participants could use auditory cues to increase swing speeds and develop ideal kinetic chains in their upper limbs, but not their trunks. Our results suggest that our proposed auditory BF system is, in part, effective and feasible for training novice baseball players in batting. Long-term studies are necessary to determine the proposed auditory BF system's exact efficiency in novice baseball players.

\section{Abbreviations \\ BF: biofeedback; PC: personal computer.}

\section{Authors' contributions}

TO and HI constructed the study concept and design. TO and SK collected data. TO and KY analyzed data and prepared the draft manuscript. All 
members verified the content of their contributions. All authors read and approved the final manuscript.

\section{Author details}

${ }^{1}$ Graduate School of Creative Science and Engineering, Waseda University, 3-4-1 Okubo, Shinjuku-ku, Tokyo 169-8555, Japan. ${ }^{2}$ Research Institute for Science and Engineering, Waseda University, 3-4-1 Okubo, Shinjuku-ku, Tokyo 169-8555, Japan.

\section{Acknowledgements}

This study was supported by the Institute of Advanced Active Aging Research.

\section{Competing interests}

The authors declare that they have no competing interests.

\section{Ethics approval and consent to participate}

This experiment was performed with approval of the Waseda University Ethical Review Board. This examination's procedure was approved by the Waseda University Ethical Review Board.

\section{Publisher's Note}

Springer Nature remains neutral with regard to jurisdictional claims in published maps and institutional affiliations.

Received: 24 January 2018 Accepted: 8 May 2018

Published online: 23 May 2018

\section{References}

1. Fleisig GS, Kwon YH (2011) Editorial. Sport Biomech 10(4):269

2. Welch CM, Banks SA, Cook FF, Draovitch PD (1995) Hitting a baseball: a biomechanical description. J Orthop Sports Phys Ther 22(5):193-20

3. Kibler WB, Wilkes T, Sciascia A (2013) Mechanics and pathomechanics in the overhand athlete. Clin Sports Med 32(4):637-651. https://doi. org/10.1016/j.csm.2013.07.003

4. Sciascia A, Thigpen C, Namdari S, Baldwin K (2012) Kinetic chain abnormalities in the athletic shoulder. Sports Med Arthrosc Rev 20(1):16-21. https://doi.org/10.1097/JSA.0b013e31823a021f

5. Ahmadi A, Rowlands D, James DA (2009) Towards a wearable device for skill assessment and skill acquisition of a tennis player during the first serve. Leis Loisir 2(3-4):129-136

6. Connaghan D, Kelly P, O'Connor NE, Gaffney M, Walsh M, O'Mathuna C (2011) Multi-sensor classification of tennis strokes. In: Proceedings of the IEEE Sensors, pp 1437-1440

7. Lai DT, Hetchl M, Wei X, Ball K, Mclaughlin P (2011) On the difference in swing arm kinematics between low handicap golfers and non-golfers using wireless inertial sensors. Proc Eng 13:219-225

8. Koda H, Sagawa K, Kuroshima K, Tsukamoto T, Urita K, Ishibashi Y (2010) 3D measurement of forearm and upper arm during throwing motion using body mounted sensor. J Adv Mech Des Syst Manuf 4(1):167-178

9. Ghasemzadeh H, Loseu V, Jafari R (2009) Wearable coach for sport training: a quantitative model to evaluate wrist-rotation in golf. J Ambient Intell Smart Environ 1(2):173-184

10. Ghasemzadeh $\mathrm{H}$, Jafari $\mathrm{R}$ (2011) Coordination analysis of human movements with body sensor networks: a signal processing model to evaluate baseball swings. Sensors J IEEE 11(3):603-610

11. Ki KI, Kim MS, Moon Y, Choi JD (2015) Effects of auditory feedback during gait training on hemiplegic patients' weight bearing and dynamic balance ability. J Phys Ther Sci 27(4):1267-1269

12. Schauer M, Mauritz KH (2003) Musical motor feedback (MMF) in walking hemiparetic stroke patients: randomized trials of gait improvement. Clin Rehabil 17(7):713-722
13. Owaki D, Sekiguchi DY, Honda K, Ishiguro A, Izumi S (2016) Shortterm effect of prosthesis transforming sensory modalities on walking in stroke patients with hemiparesis. Neural Plast. https://doi. org/10.1155/2016/6809879

14. Miyake Y (2009) Interpersonal synchronization of body motion and the walk-mate walking support robot. IEEE Trans Robot 25(3):638-644

15. Baram Y, Aharon-Peretz J, Badarny S, Susel Z, Schlesinger I (2016) Closedloop auditory feedback for the improvement of gait in patients with Parkinson's disease. J Neurol Sci 363:104-106

16. Yasuda K, Kaibuki N, Harashima H, Iwata H (2017) The effect of a haptic biofeedback system on postural control in patients with stroke: an experimental pilot study. Somatosens Mot Res 34(2):65-71

17. Saichi K, Yasuda K, Kitaji Y, Kaibuki N, Iwata H (2016) Development and pilot clinical evaluation of a haptic — based perception-empathy biofeedback device for gait rehabilitation. In: 2016 IEEE 38th annual international conference of the engineering in medicine and biology society, pp 6158-6161

18. Yasuda K, Saichi K, Kitaji Y, Harashima H, Iwata H (2017) Development of an implicit method for directing weight shifting to the affected side in patients with stroke: a proof of concept study. ROBOMECH J 4(1):26

19. Cesarini D, Calvaresi D, Farnesi C, Taddei D, Frediani S, Ungerechts BE, Hermann T (2016) Mediation: an emdedded system for auditory feedback of hand-water interaction while swimming. Proc Eng 147:324-329

20. Ronsse R, Puttemans V, Coxon JP, Goble DJ, Wagemans J, Wenderoth N, Swinnen SP (2011) Motor learning with augmented feedback: modality-dependent behavioral and neural consequences. Cereb Cortex 21(6):1283-1294

21. Shinoda S, Hashiba M (2008) Melody road and design program of melody road [Translated from Japanese]. WO2006/030915

22. Takagi T, Fujii N, Koike S, Ae K (2008) The kinematic characteristics of swing motion to the different speed balls in baseball batting [Translated from Japanese]. J Soc Biomech 32(3):158-166

23. Sarpeshkar V, Mann DL, Spratford W, Abernethy B (2017) The influence of ball-swing on the timing and coordination of a natural interceptive task. Hum Mov Sci 54:82-100

24. Ae K, Koike S, Kawamura T (2014) Kinetic analysis of upper limbs during baseball tee-batting motion at different hitting-point heights [Translated from Japanese]. Japan J Phys Educ Health Sport Sci 59(2):431-452

25. Ae K, Koike S, Kawamura T (2015) Kinetic analysis of the trunk in baseball tee-batting motion at different hitting-point heights [Translated from Japanese]. Japan J Phys Educ Health Sport Sci 60(2):635-649

26. Miyanishi T, Endo S (2016) A biomechanical study of timing baseball batting against a pitched fastball: comparison of batting situations with and without prior knowledge of the type of pitch. In: Proceeding of the 34th internationalconferenceonbiomechanics insports, Tsukuba, Japan, July 18-22, pp 839-842

27. Scott S, Gray R (2010) Switching tools: perceptual-motor recalibration to weight changes. Exp Brain Res 201(2):177-189

28. Schimidt RA (1991) Motor learning \& performance: from principles to practice. Human Kinetics Publishers Inc, Champaign

29. Aimola L, Schindler I, Simone AM, Venneri A (2012) Near and far space neglect: task sensitivity and anatomical substrates. Neuropsychologia 50(6):1115-1123

30. Wolpert DM, Miall RC, Kawato M (1998) Internal models in the cerebellum. Trends Cogn Sci 2(9):338-347

31. Koike S, Mimura K (2016) Main contributors to the baseball bat head speed considering the generating factor of motion-dependent term. In: 11 th conference of the International Sports Engineering Association, vol 147, pp 197-202

32. Koike S, Mimura K (2016) Effective timing of exerting joint torques to obtain baseball bat head speed. In: Proceedings of the 34th international conference on biomechanics in sports, pp 703-706 